Original

\title{
Análisis de la variación de la calidad de leche en Colombia 2008-2019
}

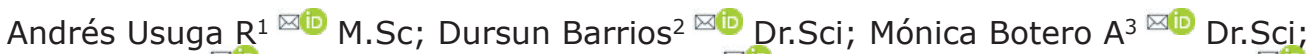 \\ Mauricio Lopera $C^{4} \bowtie\left(\mathbb{0}\right.$ M.Sc; Martha Olivera-Angel ${ }^{凶}$ Dr.Sci.Agr; Luis G Palacio $B^{1 *}{ }^{凶}$ Dr.Sci.
}

\begin{abstract}
${ }^{1}$ Universidad de Antioquia, Facultad de Ciencias Agrarias, Escuela de Medicina Veterinaria, Grupo de Investigación Biogénesis. Medellín, Colombia.

2Universidad Nacional de Colombia sede Bogotá, Facultad de Ciencias Agrarias, Departamento de Desarrollo Rural y Agroalimentario, Grupo de Investigación Biogénesis. Bogotá D.C., Colombia.

${ }^{3}$ Universidad de Antioquia, Facultad de Ciencias Agrarias, Escuela de Producción Agropecuaria, Grupo de Investigación Biogénesis. Medellín, Colombia.

${ }^{4}$ Universidad de Antioquia, Facultad de Ciencias Económicas, Departamento de Matemáticas y Estadística. Medellín, Colombia.

*Correspondencia: guillermo.palacio@udea.edu.co
\end{abstract}

Recibido: Abril 2020; Aceptado: Diciembre 2020; Publicado: Marzo 2021.

\section{RESUMEN}

Objetivo. Cuantificar los cambios en la calidad higiénica y composicional de la leche acopiada de manera formal en Colombia entre 2008 y 2019 asociados a la expedición y entrada en vigencia de la normatividad asociada a pago por calidad de leche cruda Materiales y métodos. Se utilizaron 144 reportes mensuales de calidad higiénica y composicional de la leche acopiada de manera formal para cada una de las regiones productoras de leche de Colombia consolidados por la USP-MADR. Con estos reportes se formaron tres períodos de estudio separados por la entrada en vigencia de las resoluciones 017 de 2012 y 468 de 2015. Los datos obtenidos fueron analizados bajo el esquema de regresión cuantílica. Resultados. Se encontraron diferencias entre los periodos analizados tanto para los parámetros de calidad higiénica y calidad composicional en cada una de las regiones. La calidad higiénica representó la variación más alta siendo más evidente en la región 2 . Se evidenciaron diferencias en calidad composicional de la leche acopiada de manera formal en Colombia entre los periodos asociados a la entrada en vigencia de la normatividad, principalmente en la región 1. Conclusiones. Se evidenció que la entrada en vigencia de la normatividad ejerció influencia sobre los parámetros de calidad higiénica y composicional de la leche acopiada de manera formal en Colombia.

Palabras clave: Grasa de la leche; higiene de la leche; inocuidad alimentaria; leche cruda; normas alimentarias; producción lechera; proteínas de la leche (Fuentes: AIMS, CAB).

\section{ABSTRACT}

Objective. by regions in the hygienic and compositional quality of milk collected in a formal way in Colombia between 2008-2019 associated with the issuance and entry into force of regulatory regulations. Materials and methods. 144 monthly reports on the hygienic and compositional quality of the milk collected formally were used for each of the milk-producing regions of Colombia consolidated by the USP-MADR. With these reports, three study periods were formed, separated by the entry into force of resolutions 017 of 2012 and 468 of 2015 . The data obtained was analyzed

Como citar (Vancouver).

Usuga A, Barrios D, Botero M, Lopera M, Olivera-Angel M, Palacio LG. Análisis de la variación de la calidad de leche en Colombia 2008-2019. Rev MVZ Córdoba. 2021 26(2):e2005. https://doi.org/10.21897/rmvz.2005 
under the quantile regression scheme. Results. Differences were found between the analyzed periods for the parameters of hygienic quality and compositional quality in each region. Hygienic quality represented the highest variation, being more evident in region 2 . There were differences in the compositional quality of milk collected formally in Colombia between the periods associated with the entry into force of the regulations, mainly in region 1. Conclusions. It was evidenced that the entry into force of the regulations exerted influence on the parameters of hygienic and compositional quality of milk collected formally in Colombia.

Keywords: Food safety; food standards; milk fat; milk hygiene; milk production; milk protein; raw milk (Source: $A I M S, C A B$ ).

\section{INTRODUCCIÓN}

El acopio formal de leche en Colombia se estima en 9 millones de litros por día, cerca del $47 \%$ del total de producción de leche de ganado bovino a nivel nacional. Colombia es el tercer país productor de leche en Suramérica y casi la totalidad de su producción se destina a satisfacer la demanda interna, que representa un consumo per cápita de 143 litros de leche /año $(1,2)$.

La industria láctea es cada vez más exigente con la calidad de la leche que acopia. Esta calidad es medida bajo tres grandes componentes: i. calidad sanitaria, hace referencia a la aplicación de buenas prácticas ganaderas que incluyen, vacunación contra fiebre aftosa y brucelosis y la certificación como hatos libres de brucelosis y tuberculosis bovina. ii. calidad higiénica está dada por la medición de Unidades Formadoras de Colonia (UFC) y iii. Por último, la calidad composicional mide el contenido de grasa, proteína y sólidos totales de la leche $(3,4,5,6,7)$. Estos parámetros contribuyen a determinar la inocuidad, el valor nutricional de la leche y el rendimiento cuando se elaboran derivados lácteos $(8,9)$.

Los estándares de calidad higiénica y composicional que debe cumplir la leche cruda en Colombia están definidos en los decretos 616 de 2006 y 1880 de 2011. Según éstos, la leche debe tener como mínimo $2.9 \%$ de proteína, $3.0 \%$ de grasa, $11.30 \%$ de sólidos totales y las características microbiológicas medidas en recuento de mesófilos aerobios no deben sobrepasar las $700.000 \mathrm{UFC} / \mathrm{ml}$ (5). Las resoluciones 012 de 2007 y la 017 de 2012 emitidas por el MADR se basan en esos parámetros para reglamentar los sistemas de pago por calidad de la leche cruda al productor, así como para reglamentar la conformación, habilitación y acreditación de una red de laboratorios para el análisis $(10,11)$.
En términos normativos, uno de los avances presentados en Colombia en los últimos 13 años, es la obligación que tienen los agentes compradores de leche cruda de pagarla con base en la evaluación de la calidad higiénica y composicional realizada en un laboratorio (10). El sistema de pago por calidad y la acreditación de la red de laboratorios aseguran la transparencia en el pago de la leche.

Inicialmente, desde febrero de 2007, la determinación de la calidad de la leche debía ser efectuada por un laboratorio habilitado por Corpoica (ahora Agrosavia). En el año 2015 el MADR expidió la resolución 468 que reiteró el carácter obligatorio de la evaluación de la calidad higiénica y composicional de la leche cruda pero esta vez, el análisis debía ser realizado por laboratorios acreditados en NTC-ISO/IEC 17025 ante el Organismo Nacional de Acreditación de Colombia- ONAC y estableció el 01 de agosto de 2016 como fecha límite para la acreditación de los laboratorios (10). Con la acreditación en la norma NTC-ISO/IEC 17025 se logra el cumplimiento de requisitos de calidad y su mejoramiento continuo para alcanzar los más altos niveles de reconocimiento, no solo a nivel nacional sino también a nivel internacional. (12).

En Colombia, para el 10 agosto del año 2020 había siete laboratorios acreditados por el ONAC para procesar muestras de leche y determinar calidad asociada a pago, tres de ellos independientes (dos de Agrosavia y uno de la Universidad de Antioquia) y cuatro pertenecientes a la industria acopiadora de leche (Alpina, Colanta, Freskaleche y Nestlé) (13).

La metodología para obtener el precio pagado al productor, se basa en los resultados entregados por los laboratorios acreditados en términos de calidad higiénica y composicional de la leche. El análisis de éstos permite entender las variaciones del precio pagado al productor, regulado mediante la resolución 017 de 2012, 
según la cual el pago debe ser calculado con base en el contenido de proteína y grasa o el contenido de sólidos totales por litro de leche y además, bonificación o castigo por calidad higiénica medida en UFC/ml (10).

El objetivo de esta investigación fue cuantificar los cambios en la calidad higiénica y composicional de la leche acopiada de manera formal en Colombia asociados a la expedición y entrada en vigencia de las resoluciones regulatorias de pago de leche cruda 012/2007, 017/2012 y 468/2015.

\section{MATERIALES Y MÉTODOS}

Tipo de estudio. Se realizó un estudio observacional descriptivo retrospectivo basado en series de tiempo interrumpidas de calidad higiénica y composicional de leche cruda acopiada formalmente entre enero 2008 y diciembre 2019. La investigación está basada en el análisis de fuentes secundarias de datos sobre calidad higiénica y composicional de leche cruda acopiada de manera formal en Colombia.

Área de estudio. Este estudio se llevó a cabo con información generada en los departamentos de Colombia donde se realiza acopio formal de leche. Según La resolución 017/2012 del Ministerio de Agricultura y Desarrollo Rural de Colombia se definió el pago diferencial de la leche por regiones lecheras (región 1 y 2), que corresponden a un conjunto de departamentos que comparten características similares desde el punto de vista productivo (Figura 1) (10).

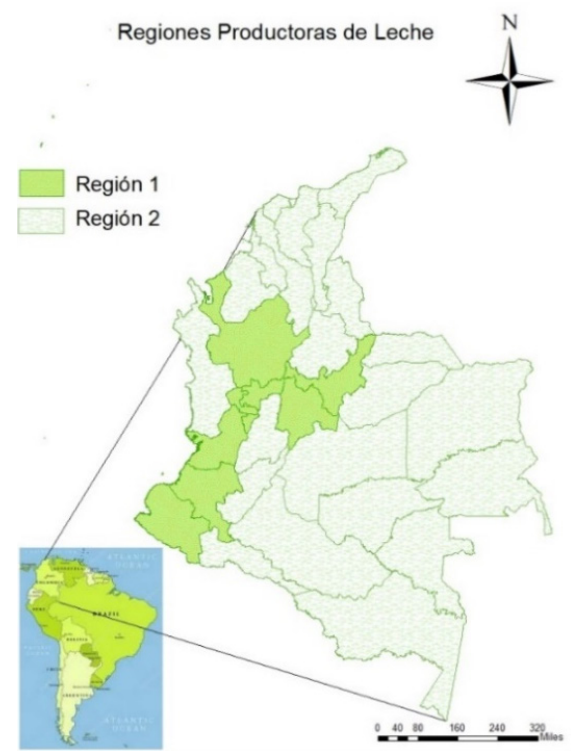

Figura 1. Clasificación de Colombia por regiones productoras de leche.
Métodos de obtención de datos sobre reportes de calidad higiénica y composicional. Los agentes compradores de leche reportan los datos de calidad microbiológica y composicional ponderados por volumen de leche acopiado a la USP-MADR quincenalmente. La USP-MADR consolida y entrega la información por departamento, región y nacional, también ponderada por volumen. Esta información se encuentra publicada en la página web de la USP- MADR (1).

Con los datos obtenidos, se realizó un análisis estadístico para determinar el posible efecto de la entrada en vigencia de las tres normas relacionadas con los reportes de calidad de la leche que se acopió de manera formal durante el periodo comprendido entre enero 2008 y diciembre de 2019.

Variables y nivel de medición. Se incluyó la variable acopio formal, expresada en millones de litros de leche por mes. También se tomó la variable calidad microbiológica, expresada en $\mathrm{UFC} / \mathrm{ml}$ y además se incluyeron las variables de calidad composicional: i. Porcentaje de grasa en leche, ii. Porcentaje de proteína en leche y iii. Porcentaje de sólidos totales. Estas variables tienen una periodicidad de reporte mensual en el portal de la USP-MADR.

Análisis de la información. Para establecer la relación entre las variables de calidad higiénica y composicional con la entrada en vigencia de las resoluciones 012 de 2007, 017 del 2012 y 468 de 2015 se construyeron series de tiempo interrumpidas. Se utilizaron 144 reportes mensuales de calidad higiénica y composicional de la leche acopiada de manera formal por los agentes compradores en cada una de las regiones y consolidados por la USP-MADR.

Los datos obtenidos fueron analizados bajo el esquema de regresión cuantílica. Se formaron tres períodos de estudio así: 1. desde el 01 de enero de 2008 posterior a la entrada en vigencia de la resolución 012 de enero de 2007, hasta el 28 de febrero de 2012; 2. desde 01 de marzo de 2012, cuando entra en vigencia de la resolución 017 de 2012 hasta el 31 de julio de 2016 y 3 . desde el 01 de agosto de 2016, con la entrada en vigencia de la resolución 468 de 2015, hasta el 31 de diciembre de 2019. El periodo de observación no incluyó información de 2007 pues esta no estaba disponible en la USP-MADR $(10,11)$. 
Análisis estadístico. Las variables de calidad composicional de la leche presentan patrones estacionales. Por esta razón el análisis de la entrada en vigencia de las resoluciones 012/2007, 017/2012 y 468/2015 debía incluir de forma simultánea variables que tuvieran en cuenta los patrones estacionales. Con este fin, en este trabajo se incluyeron doce variables categóricas que representaron el efecto estacional de cada mes del año sobre cada una de las variables de calidad composicional.

El modelo econométrico usado para cada una de las variables de calidad composicional de la leche es el que se presenta para proteína, pero se aplica el mismo para las demás variables de interés:

Proteina $\mathrm{t}_{\mathrm{t}}=\mathrm{B}_{1} \mathrm{~d}_{1, \mathrm{t}}+\mathrm{B}_{2} \mathrm{~d}_{2, \mathrm{t}}+\mathrm{B}_{3} \mathrm{~d}_{3, \mathrm{t}}+\mathrm{B}_{4} \mathrm{~d}_{4, \mathrm{t}}+\mathrm{B}_{5} \mathrm{~d}_{5}$ ${ }_{, t}+B_{6} d_{6, t}+B_{7} d_{7, t}+B_{8} d_{8, t}+B_{9} d_{9, t}+B_{10} d_{10, t}+B_{11}$ $\mathrm{d}_{11, \mathrm{t}}+\mathrm{B}_{12} \mathrm{~d}_{12, \mathrm{t}}+\propto_{2} \mathrm{~m}_{2, \mathrm{t}}+\propto_{3} \mathrm{~m}_{3, \mathrm{t}}+\epsilon_{\mathrm{t}}$

$\mathrm{d}_{\mathrm{i}, \mathrm{t}}=1$, si $\mathrm{t} \in$ mes i del año 0 , si no $i=1,2, \ldots, 12$

$\mathrm{m}_{\mathrm{j}, \mathrm{t}}=1$, si $\mathrm{t} \in$ al periodo $\mathrm{j} 0$, si no $j=2,3$

$\propto_{2} \mathrm{y} \propto_{3}$ representan el efecto de las resoluciones $07 / 2012$ y $468 / 2015$ respectivamente, manteniendo lo demás constante

Dado que no hay variable categoría para el periodo enero 2008- febrero 2012 en el modelo, este periodo queda como categoría de referencia.

El Modelo para la categoría de referencia es $\left(m_{2, \mathrm{t}}=0\right.$ y $\left.\mathrm{m}_{3, \mathrm{t}}=0\right)$

Proteina $\mathrm{t}_{\mathrm{t}}=\mathrm{B}_{1} \mathrm{~d}_{1, \mathrm{t}}+\mathrm{B}_{2} \mathrm{~d}_{2, \mathrm{t}}+\mathrm{B}_{3} \mathrm{~d}_{3, \mathrm{t}}+\mathrm{B}_{4} \mathrm{~d}_{4, \mathrm{t}}+\mathrm{B}_{5} \mathrm{~d}_{5}$ ,t $+B_{6} d_{6, t}+B_{7} d_{7, t}+B_{8} d_{8, t}+B_{9} d_{9, t}+B_{10} d_{10, t}+B_{11}$ $\mathrm{d}_{11, \mathrm{t}}+\mathrm{B}_{12} \mathrm{~d}_{12, \mathrm{t}}+\epsilon_{\mathrm{t}}$

El modelo para el periodo marzo 2012-julio 2016 está dado por $\left(m_{2, t}=1\right.$ y $\left.m_{3, t}=0\right)$

Proteina $\mathrm{t}_{\mathrm{t}}=\mathrm{B}_{1} \mathrm{~d}_{1, \mathrm{t}}+\mathrm{B}_{2} \mathrm{~d}_{2, \mathrm{t}}+\mathrm{B}_{3} \mathrm{~d}_{3, \mathrm{t}}+\mathrm{B}_{4} \mathrm{~d}_{4, \mathrm{t}}+\mathrm{B}_{5} \mathrm{~d}_{5}$ ,$t+B_{6} d_{6, t}+B_{7} d_{7, t}+B_{8} d_{8, t}+B_{9} d_{9, t}+B_{10} d_{10, t}+B_{11}$ $\mathrm{d}_{11, \mathrm{t}}+\mathrm{B}_{12} \mathrm{~d}_{12, \mathrm{t}}+\propto_{2}+\epsilon_{\mathrm{t}}$

Y por último, el modelo a partir de agosto de 2016 es $\left(m_{2, t}=0\right.$ y $\left.m_{3, t}=1\right)$

Proteina $\mathrm{t}_{\mathrm{t}}=\mathrm{B}_{1} \mathrm{~d}_{1, \mathrm{t}}+\mathrm{B}_{2} \mathrm{~d}_{2, \mathrm{t}}+\mathrm{B}_{3} \mathrm{~d}_{3, \mathrm{t}}+\mathrm{B}_{4} \mathrm{~d}_{4, \mathrm{t}}+\mathrm{B}_{5} \mathrm{~d}_{5}$ , $t+B_{6} d_{6, t}+B_{7} d_{7, t}+B_{8} d_{8, t}+B_{9} d_{9, t}+B_{10} d_{10, t}+B_{11}$ $\mathrm{d}_{11, \mathrm{t}}+\mathrm{B}_{12} \mathrm{~d}_{12, \mathrm{t}}+\propto_{3}+\epsilon_{\mathrm{t}}$
Debido a que este modelo estima efectos parciales promedio, estos últimos pueden estar influenciados por datos atípicos y esquemas heterocedásticos. Con el fin de evitar su posible influencia, se recurre al esquema de regresión cuantil, que al estudiar de forma completa la distribución de probabilidad de la variable, corrige la situación de datos atípicos y los posibles esquemas heterocedásticos que presentan los modelos de regresión (14). Las estimaciones de este modelo se hicieron para la mediana, es decir, el cuantil 50, medida de tendencia central que no es influenciada por la presencia de posibles valores extremos. Los datos fueron tabulados en libro de Excel y analizados con el software libre y de código abierto $R$.

Aspectos éticos. Según la resolución 8430 de 1993 del Ministerio de Salud de Colombia "Por la cual se establecen las normas científicas, técnicas y administrativas para la investigación en salud" este estudio se enmarca en la categoría de investigación sin riesgo. Los datos de este estudio fueron obtenidos de revisión documental retrospectiva, no se realizó ninguna modificación intencionada a variables biológicas o fisiológicas y no se contó con la participación de seres vivos. Dadas estas características no se dio la necesidad de realizar consentimiento informado ni sometimiento a comité de ética.

\section{RESULTADOS}

Volumen de acopio formal. En el Figura 2 se presentan la tendencia de acopio formal de leche en Colombia durante el periodo 2008-2019 (Figura 2A) y los resultados del modelo para la comparación de las medianas por periodo del volumen mensual de acopio. La región 1 mostró un aumento progresivo en el acopio y diferencias significativas entre periodos $(p<0.001)$ (Figura 2B). Para el caso de la región 2, el análisis por periodos mostró un aumento en el volumen de acopio para el periodo 2 respecto al periodo 1 (Figura 2C). Sin embargo, el volumen de acopio disminuyó para el periodo 3, cuando se comparó con el periodo 1. Es evidente la estacionalidad en el volumen de acopio formal que se presentó en la región 2 , siendo mayor la cantidad de leche en los meses de mayo a octubre; entretanto los meses de febrero-abril registraron los volúmenes de acopio más bajos. 

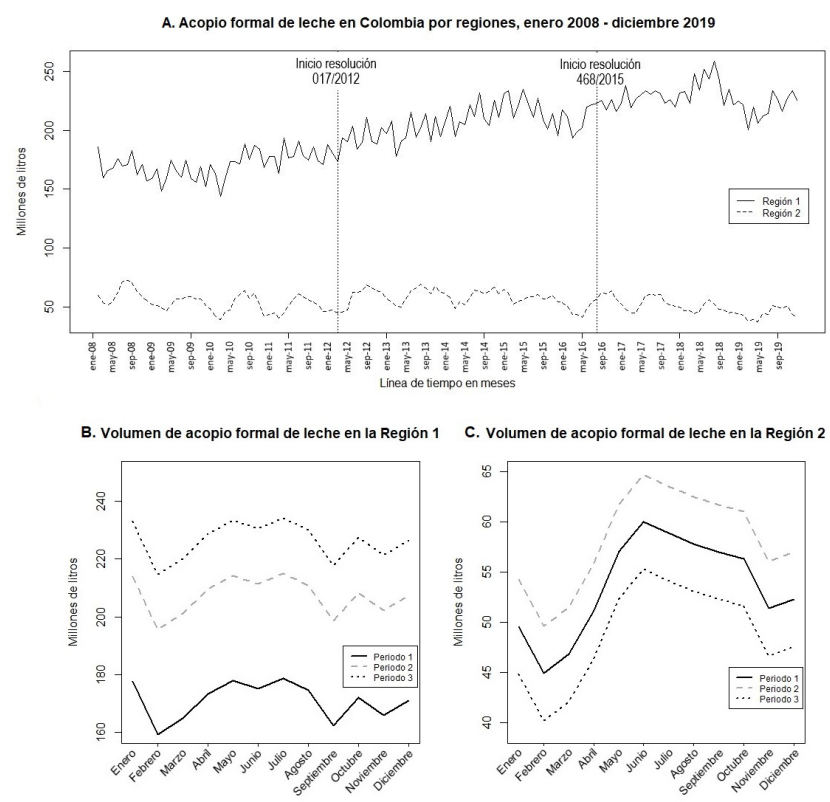

Figura 2. Serie de tiempo y medianas de acopio formal de leche cruda en Colombia por periodo y región, según los meses del año, 2008 -2019.

Unidades Formadoras de Colonia (UFC). La serie de tiempo de las UFC reportadas para la leche acopiada de manera formal en la región 1 muestra una tendencia estable (Figura 3A) En las figuras $3 \mathrm{~B}$ y $3 \mathrm{C}$ se muestra cómo cambian las medianas de UFC en los tres períodos durante los distintos meses del año, para cada mes los coeficientes $B_{i}$ para el primer periodo, $\mathrm{B}_{\mathrm{i}}+\propto_{2}$ para el segundo período y $\mathrm{B}_{\mathrm{i}}+\propto_{3}$ para el tercer periodo. Con base en esta información y de la significancia estadística $(p<0.01)$ de los coeficientes $\propto_{2}$ y $\propto_{3}$ se puede concluir que las resoluciones $017 / 2012$ y 468/2015 estuvieron asociadas a la modificación en las UFC reportadas en la leche que es acopiada de manera formal tanto en la región 1 como en la región 2 .

Al realizar la comparación entre regiones, se observó que los promedios de UFC/ml reportados para la región 2 fueron dos veces aquellos reportados para la región 1 . Es necesario destacar la marcada disminución de las UFC de la leche acopiada en la región 2, particularmente durante el periodo 2 (Figura 3A).

Los errores estándar de cada uno de los coeficientes fueron estimados mediante la técnica Bootstrap con 20000 replicaciones. Es de notar que todos los coeficientes son estadísticamente significativos para la región 1 $(p<0.001)$. Además, este modelo se interpreta en las variables $m_{2, t}$ y $m_{3, t}$ con intercepto variante con respecto al mes. De esta forma, la mediana estimada para la variable UFC en el mes 1 está dada por 464865 en el periodo 1 ; 464865-89689 para el período 2 y $464865+87087$ para el período 3.

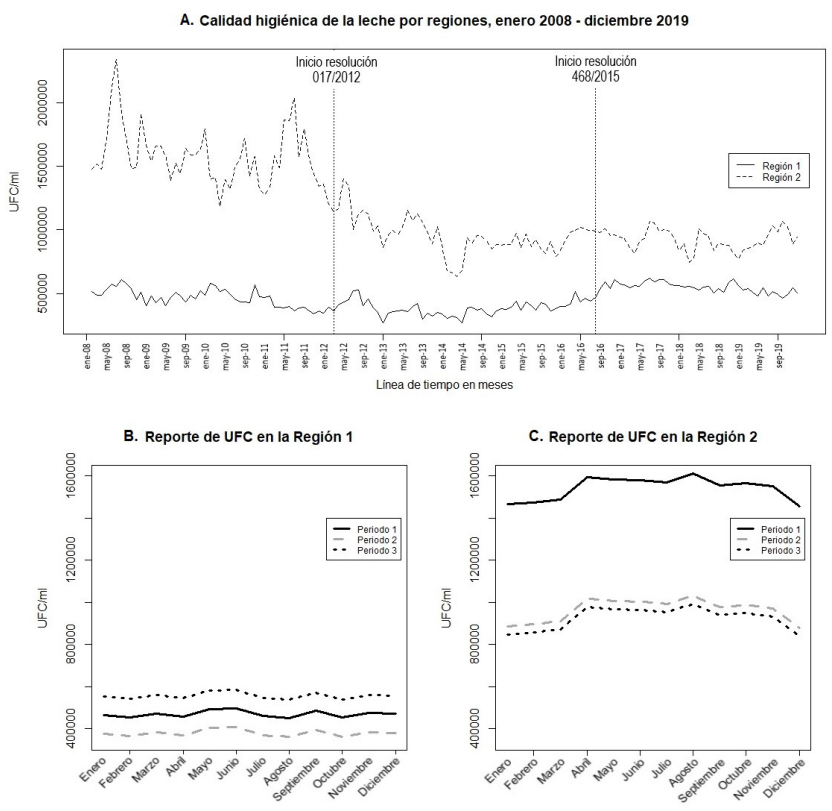

Figura 3. Serie de tiempo y distribución por cuantiles de las UFC reportadas por regiones para la leche acopiada de manera formal en Colombia, 2008 - 2019

En la región 1, en el periodo 2 hay una reducción significativa de UFC respecto al periodo 1 $(p<0.001)$. Sin embargo, en el periodo 3 hay un incremento con respecto a los demás periodos $(p<0.001)$. Dada la significancia de $\propto_{2} y \propto_{3}$ se puede afirmar que la entrada en vigencia de las resoluciones $017 / 2012$ y $468 / 2015$ estuvieron asociadas a un cambio en las UFC de la leche acopiada de manera formal en la región 1 (Tabla 1).

Para el caso de la región 2, se encontró que todos los coeficientes son estadísticamente significativos $(p<0.001)$. Al considerar los interceptos variantes respecto al mes, se tiene que la mediana estimada para la variable UFC de la leche acopiada de manera formal en la región 2 en el mes 1 está dada por 1465576 en el periodo $1 ;$ 1465576-578339 para el periodo 2 y $1465576-617274$ para el periodo 3 . En los periodos 2 y 3 hay reducciones estadísticamente significativas respecto al periodo 1 . Dada la significancia de $\propto_{2}$ y $\propto_{3}$ Se tiene entonces que, la entrada en vigencia de las resoluciones 017/2012 y 468/2015 tuvieron un efecto positivamente significativo sobre el reporte de UFC en la leche acopiada de manera formal en la región 2 (Tabla 1). 
Tabla 1. Modelos estimados para las variables acopio, UFC, proteína, grasa y sólidos totales de la leche que se acopia de manera formal en las regiones 1 y 2 para la regresión mediana, para el periodo 1.

\begin{tabular}{|c|c|c|c|c|c|c|c|c|c|}
\hline \multirow{2}{*}{ Variable } & \multirow{2}{*}{ Mes } & \multicolumn{4}{|c|}{ Región 1} & \multicolumn{4}{|c|}{ Región 2} \\
\hline & & Mediana & Error estándar & Valor $\mathbf{t}$ & Valor $\mathbf{p}$ & Mediana & Error estándar & Valor t & Valor $\mathbf{p}$ \\
\hline \multirow{14}{*}{ Acopio } & Enero & 177.710 .590 & 4.30 & 41.34 & 0.00 & 49.581 .510 & 2.23 & 22.20 & 0.00 \\
\hline & Febrero & 159.473 .800 & 5.36 & 29.76 & 0.00 & 44.934 .400 & 2.36 & 19.05 & 0.00 \\
\hline & Marzo & 164.927 .900 & 5.52 & 29.86 & 0.00 & 46.828 .640 & 1.74 & 26.99 & 0.00 \\
\hline & Abril & 173.462 .410 & 4.88 & 35.50 & 0.00 & 51.181 .810 & 2.50 & 20.45 & 0.00 \\
\hline & Mayo & 178.080 .990 & 3.77 & 47.28 & 0.00 & 57.100 .640 & 1.81 & 31.49 & 0.00 \\
\hline & Junio & 175.183 .260 & 5.67 & 30.91 & 0.00 & 60.046 .910 & 2.29 & 26.28 & 0.00 \\
\hline & Julio & 178.714 .020 & 5.53 & 32.29 & 0.00 & 58.882 .910 & 2.07 & 28.41 & 0.00 \\
\hline & Agosto & 174.733 .280 & 2.91 & 59.81 & 0.00 & 57.824 .750 & 2.81 & 20.58 & 0.00 \\
\hline & Septiembre & 162.476 .870 & 3.71 & 43.8 & 0.00 & 57.002 .190 & 2.28 & 25.02 & 0.00 \\
\hline & Octubre & 172.121 .400 & 3.06 & 56.3 & 0.00 & 56.348 .620 & 2.27 & 24.82 & 0.00 \\
\hline & Noviembre & 166.030 .550 & 4.06 & 40.88 & 0.00 & 51.390 .490 & 2.92 & 17.61 & 0.00 \\
\hline & Diciembre & 171.166 .420 & 4.16 & 41.11 & 0.00 & 52.300 .220 & 2.51 & 20.80 & 0.00 \\
\hline & $\alpha 2$ & 36,17 & 3.04 & 11.88 & 0.00 & 4.68 & 1.32 & 3.55 & 0.00 \\
\hline & $\alpha 3$ & 55,19 & 2.59 & 21.31 & 0.00 & -4.75 & 1.78 & -2.67 & 0.01 \\
\hline \multirow{14}{*}{ UFC } & Enero & 464.685 & 22.411 & 20.74 & 0.00 & 1.465 .576 & 54.545 & 26.86 & 0.00 \\
\hline & Febrero & 454.182 & 24.578 & 18.47 & 0.00 & 1.474 .903 & 63.962 & 23.05 & 0.00 \\
\hline & Marzo & 471.983 & 23.267 & 20.28 & 0.00 & 1.489 .002 & 61.884 & 24.06 & 0.00 \\
\hline & Abril & 458.220 & 34.491 & 13.28 & 0.00 & 1.595 .434 & 70.515 & 22.62 & 0.00 \\
\hline & Mayo & 492.888 & 25.251 & 19.59 & 0.00 & 1.584 .643 & 93.717 & 16.90 & 0.00 \\
\hline & Junio & 497.934 & 25.912 & 19.21 & 0.00 & 1.581 .181 & 68.745 & 23.00 & 0.00 \\
\hline & Julio & 459.606 & 33.085 & 13.89 & 0.00 & 1.569 .017 & 60.997 & 25.72 & 0.00 \\
\hline & Agosto & 451.246 & 25.197 & 17.91 & 0.00 & 1.610 .667 & 57.598 & 27.96 & 0.00 \\
\hline & Septiembre & 485.547 & 37.234 & 13.03 & 0.00 & 1.555 .442 & 51.174 & 30.39 & 0.00 \\
\hline & Octubre & 452.930 & 23.799 & 19.03 & 0.00 & 1.566 .737 & 47.678 & 32.86 & 0.00 \\
\hline & Noviembre & 474.551 & 24.253 & 19.56 & 0.00 & 1.551 .080 & 77.973 & 19.89 & 0.00 \\
\hline & Diciembre & 470.650 & 28.325 & 16.61 & 0.00 & 1.457 .105 & 64.692 & 22.52 & 0.00 \\
\hline & $\alpha 2$ & -89.689 & 18.738 & -4.78 & 0.00 & -578.339 & 47.988 & -12.06 & 0.00 \\
\hline & $\alpha 3$ & 87.087 & 17.727 & 4.91 & 0.00 & -617.274 & 47.442 & -13.01 & 0.00 \\
\hline \multirow{14}{*}{ Proteína } & Enero & 3.04 & 0.04 & 76.63 & 0.00 & 3.36 & 0.02 & 155.6 & 0.00 \\
\hline & Febrero & 3.02 & 0.02 & 137.29 & 0.00 & 3.31 & 0.03 & 130.01 & 0.00 \\
\hline & Marzo & 3.02 & 0.02 & 162.84 & 0.00 & 3.29 & 0.02 & 176.97 & 0.00 \\
\hline & Abril & 3.01 & 0.02 & 159.87 & 0.00 & 3.3 & 0.02 & 179.22 & 0.00 \\
\hline & Mayo & 3.01 & 0.02 & 160.61 & 0.00 & 3.31 & 0.03 & 120.49 & 0.00 \\
\hline & Junio & 3.02 & 0.02 & 146.85 & 0.00 & 3.38 & 0.02 & 162.45 & 0.00 \\
\hline & Julio & 3.02 & 0.02 & 130.43 & 0.00 & 3.41 & 0.03 & 134.66 & 0.00 \\
\hline & Agosto & 3.02 & 0.02 & 148.29 & 0.00 & 3.41 & 0.02 & 185.85 & 0.00 \\
\hline & Septiembre & 3.03 & 0.02 & 147.87 & 0.00 & 3.45 & 0.02 & 142.07 & 0.00 \\
\hline & Octubre & 3.03 & 0.02 & 137.24 & 0.00 & 3.45 & 0.02 & 159.5 & 0.00 \\
\hline & Noviembre & 3.03 & 0.02 & 133.14 & 0.00 & 3.43 & 0.02 & 217.69 & 0.00 \\
\hline & Diciembre & 3.05 & 0.03 & 122.05 & 0.00 & 3.41 & 0.03 & 109.67 & 0.00 \\
\hline & $\alpha 2$ & 0.07 & 0.02 & 3.69 & 0.00 & -0.07 & 0.01 & -5.65 & 0.00 \\
\hline & $\alpha 3$ & 0.14 & 0.02 & 7.36 & 0.00 & -0.02 & 0.02 & -1.03 & 0.31 \\
\hline \multirow{14}{*}{ Grasa } & Enero & 3.61 & 0.04 & 86.00 & 0.00 & 3.83 & 0.06 & 66.82 & 0.00 \\
\hline & Febrero & 3.60 & 0.02 & 184 & 0.00 & 3.74 & 0.05 & 69.52 & 0.00 \\
\hline & Marzo & 3.59 & 0.03 & 134.96 & 0.00 & 3.70 & 0.04 & 94.22 & 0.00 \\
\hline & Abril & 3.56 & 0.02 & 144.83 & 0.00 & 3.69 & 0.03 & 109.40 & 0.00 \\
\hline & Mayo & 3.56 & 0.02 & 185.09 & 0.00 & 3.74 & 0.04 & 96.15 & 0.00 \\
\hline & Junio & 3.56 & 0.02 & 189.04 & 0.00 & 3.79 & 0.04 & 103.43 & 0.00 \\
\hline & Julio & 3.58 & 0.02 & 208.23 & 0.00 & 3.79 & 0.05 & 83.85 & 0.00 \\
\hline & Agosto & 3.60 & 0.02 & 181.95 & 0.00 & 3.88 & 0.10 & 37.12 & 0.00 \\
\hline & Septiembre & 3.60 & 0.02 & 189.97 & 0.00 & 3.90 & 0.08 & 47.17 & 0.00 \\
\hline & Octubre & 3.63 & 0.02 & 162.93 & 0.00 & 3.98 & 0.09 & 43.77 & 0.00 \\
\hline & Noviembre & 3.61 & 0.02 & 187.1 & 0.00 & 3.94 & 0.08 & 47.57 & 0.00 \\
\hline & Diciembre & 3.60 & 0.02 & 158.99 & 0.00 & 3.88 & 0.07 & 52.68 & 0.00 \\
\hline & $\alpha 2$ & 0.06 & 0.01 & 4.11 & 0.00 & -0.04 & 0.02 & -1.49 & 0.14 \\
\hline & $\alpha 3$ & 0.10 & 0.02 & 5.65 & 0.00 & -0.03 & 0.35 & -0.09 & 0.93 \\
\hline
\end{tabular}




\begin{tabular}{cccccccccc}
\hline \multirow{2}{*}{ Variable } & \multirow{2}{*}{ Mes } & \multicolumn{4}{c}{ Región 1 } & \multicolumn{3}{c}{ Región 2 } \\
\cline { 2 - 8 } & Mediana & Error estándar & Valor t & Valor p & Mediana & Error estándar & Valor t & Valor p \\
\hline & Enero & 11.95 & 0.02 & 641.21 & 0.00 & 12.52 & 0.04 & 309.45 & 0.00 \\
& Febrero & 11.96 & 0.03 & 409.79 & 0.00 & 12.40 & 0.10 & 130.03 & 0.00 \\
& Marzo & 11.90 & 0.03 & 455.09 & 0.00 & 12.37 & 0.05 & 273.76 & 0.00 \\
& Abril & 11.90 & 0.02 & 581.64 & 0.00 & 12.42 & 0.04 & 349.55 & 0.00 \\
& Mayo & 11.96 & 0.03 & 457.59 & 0.00 & 12.51 & 0.03 & 494.40 & 0.00 \\
Porcentaje & Junio & 11.94 & 0.02 & 574.53 & 0.00 & 12.55 & 0.04 & 301.96 & 0.00 \\
de sólidos & Julio & 11.97 & 0.03 & 390.53 & 0.00 & 12.61 & 0.03 & 503.67 & 0.00 \\
totales & Agosto & 11.96 & 0.02 & 675.70 & 0.00 & 12.62 & 0.03 & 472.09 & 0.00 \\
& Septiembre & 11.96 & 0.09 & 138.01 & 0.00 & 12.64 & 0.03 & 411.86 & 0.00 \\
& Octubre & 11.97 & 0.02 & 637.57 & 0.00 & 12.67 & 0.04 & 290.13 & 0.00 \\
& Noviembre & 11.98 & 0.02 & 672.10 & 0.00 & 12.71 & 0.03 & 438.91 & 0.00 \\
& Diciembre & 11.98 & 0.02 & 659.88 & 0.00 & 12.65 & 0.04 & 285.33 & 0.00 \\
& $\alpha 2$ & 0.10 & 0.01 & 7.58 & 0.00 & -0.01 & 0.02 & -0.44 & 0.66 \\
& $\alpha 3$ & 0.11 & 0.02 & 6.66 & 0.00 & -0.04 & 0.03 & -1.37 & 0.17 \\
\hline
\end{tabular}

Contenido de proteína en leche. En contraste con lo observado para la región 1 , en la región 2 son notorios los picos en los reportes de los meses de octubre, mostrando una marcada tendencia estacional (Figura 4A).

Las figuras 4B y 4C muestran cómo cambian las medianas de proteína en los tres períodos durante los distintos meses del año. De la gráfica 4B y la significancia estadística de los coeficientes $\propto_{2} \mathrm{Y} \propto_{3}$ se puede concluir que las resoluciones $017 / 2012$ y $468 / 2015$ tuvieron un efecto estadísticamente significativo sobre el porcentaje de proteína en la leche y que la última resolución, dado el mayor valor del coeficiente, fue la de mayor efecto cuando se evaluó la región 1 . Sin embargo, para la región 2 solo se encontraron diferencias estadísticamente significativas entre el periodo 1 y periodo 2 (Tabla 1 ).

Los errores estándar de cada uno de los coeficientes fueron estimados de la misma manera que para la UFC. Es de notar que todos los coeficientes son estadísticamente significativos para la región $1(p<0.001)$. La mediana estimada para la cantidad de proteína en el mes 1 está dada por 3.04 en el periodo 1 ; $3.04+0.06$ para el período 2 y $3.04+0.14$ para el período 3. Nótese como en el periodo 3 hay un incremento significativo con respecto a los demás periodos. Dada la significancia $\propto_{2}$ y de $\propto_{3}$ se puede afirmar, que la entrada en vigencia de las resoluciones $017 / 2012$ y $468 / 2015$ tuvieron un efecto positivamente significativo sobre el porcentaje de proteína en la leche (Tabla 1).
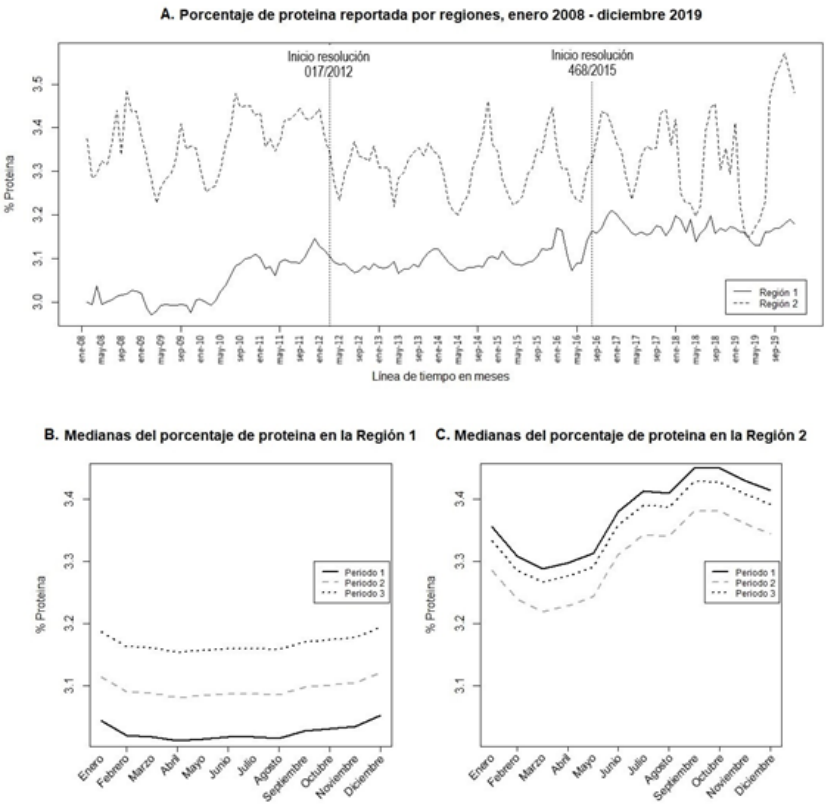

Figura 4. Serie de tiempo y distribución por cuantiles del porcentaje de proteína reportada por regiones para para la leche acopiada formalmente en Colombia, 2008 - 2019.

Para la región 2, los errores estándar de cada uno de los coeficientes fueron estimados bajo la misma técnica Bootstrap con 20000 replicaciones utilizada en los modelos anteriores. En el periodo 2 hubo una reducción significativa con respecto al periodo 1 , mientras que para el periodo 3 no se evidenció diferencias respecto al periodo de referencia. Dada la significancia $\propto_{2}$ se puede decir que la entrada en vigencia de la resolución 017/2012 tuvo un efecto negativamente significativo sobre el porcentaje de proteína en la leche (Tabla 1). 
Contenido de grasa en leche. Los reportes de porcentaje grasa en leche cumplen con los parámetros establecidos en la norma. La región 1 muestra una tendencia al aumento que es constante durante toda la observación, llegando incluso a cifras similares a las reportadas en la región 2 para el periodo 3 . Por otra parte, la región 2 mostró una marcada estacionalidad y una tendencia a la disminución (Figura 5A).

Se calcularon los errores estándar bajo la misma técnica mencionada para las UFC y la proteína. Todos los coeficientes son estadísticamente significativos para la región $1(p<0.001)$. En el periodo 3 se observa un incremento significativo con respecto a los demás periodos. Dada la significancia $\propto_{2}$ y de $\propto_{3}$ se puede afirmar, que la entrada en vigencia de las resoluciones 017/2012 y $468 / 2015$ coincidieron un efecto positivamente significativo sobre el porcentaje de grasa en la leche acopiada de manera formal en la región $1(p<0.001)$. Al aplicar la misma técnica en los datos de la región 2 no se encontraron diferencias entre periodos (Tabla 1 ).
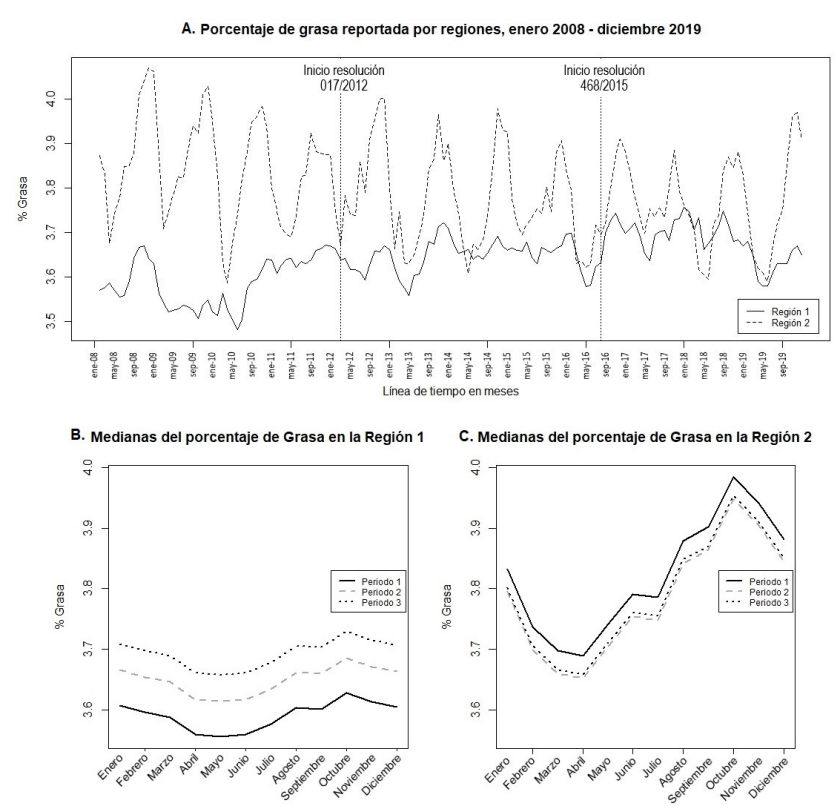

Figura 5. Serie de tiempo y distribución por cuantiles del porcentaje de grasa reportado por regiones en la leche acopiada de manera formal desde enero de 2008 a diciembre de 2019.

Contenido de sólidos totales en leche. El contenido de sólidos totales de la leche acopiada en la región 1 mostró una tendencia positiva a lo largo de toda la observación, principalmente en los periodos 1 y 2 . Además, el aumento de los porcentajes de sólidos totales en leche fue constante. En contraste con estos resultados, la región 2 mostró una marcada estacionalidad en los porcentajes de sólidos totales reportados, aunque más irregular, si se compara con la curva de proteína y grasa de la misma región (Figura 6A).

Respecto a la comparación de medianas, se encontró que en la región 1 los porcentajes de sólidos totales aumentaron de manera progresiva durante los periodos 2 y 3 . Además, los reportes fueron relativamente estables a lo largo del año (Figura 6B). Caso contrario ocurrió en la región 2 , donde no se evidenciaron diferencias significativas entre las medianas de sólidos totales reportados por periodos (Tabla 1 ). Sin embargo, si fue evidente la estacionalidad respecto a los meses del año, encontrándose que en los meses de marzo se reportaron los porcentajes más bajos de sólidos totales y en los meses de noviembre los reportes más altos (Figura 6C).
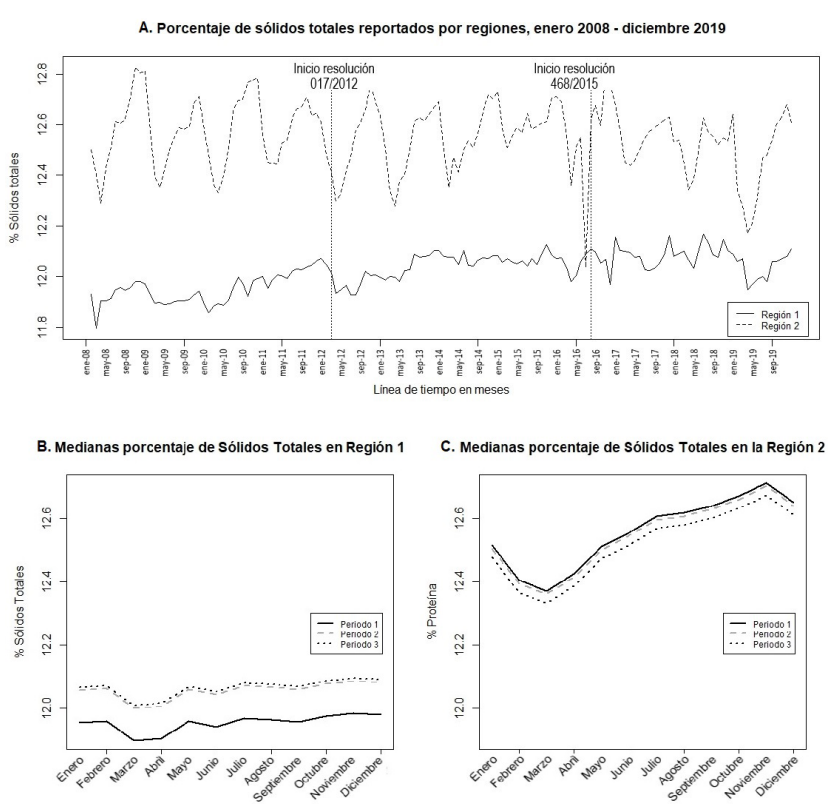

Figura 6. Serie de tiempo y distribución por cuantiles del porcentaje de Distribución por cuantiles del porcentaje de sólidos totales reportados por regiones para la leche acopiada de manera formal desde enero 2008 a diciembre de 2019.

Los modelos de regresión mediana estimados para las variables Acopio, UFC, grasa, proteína y sólidos totales de las regiones 1 y 2 en el cuantil 50 , se presentan a continuación en la tabla 1 . 


\section{DISCUSIÓN}

Colombia ha aumentado la cantidad de leche acopiada, con un incremento del $8.9 \%$ en el periodo de observación (2). Este mayor volumen se explica principalmente por el incremento de acopio de leche en la región 1 (Figura 2A).

El acopio formal de leche en la región 2 presentó variaciones dependiendo de la época del año. En los meses comprendidos entre diciembre y marzo, que coinciden con la época seca, se reportó un menor volumen de acopio de leche. El máximo volumen se reportó entre los meses de mayo y septiembre, que corresponde tradicionalmente con los meses de lluvias en dicha región. Estos resultados coinciden con lo reportado por Lambertz et al (15) quienes encontraron que variables meteorológicas como índices de temperatura y humedad influyen de manera directa sobre la producción de leche.

Por otra parte, la realización de los ensayos interlaboratorio mencionados en la resolución 017/2012 (10) coinciden con un incremento en la calidad higiénica de la leche, mucho más marcada en la región 2. Esto podría estar explicado por una mayor precisión de las metodologías diagnósticas implementadas posterior a la entrada en vigencia de las resoluciones. Desafortunadamente no se lograron encontrar referencias que soporten estas afirmaciones en el área de interés. Sin embargo, otras áreas del conocimiento tales como la salud pública han reportado mejoras en parámetros analizados, posterior a la implementación de normas específicas (16).

Las diferencias halladas entre los periodos de observación para los reportes de UFC/ml de leche acopiada de manera formal en la región 2 sugieren que la entrada en vigencia de la normatividad, contribuyó a mejorar la calidad higiénica, evidenciado al encontrar que las medianas de UFC/ml de los periodos 2 y 3 fueron menores a la mediana UFC/ml del periodo 1 , sin embargo, la información con la que se cuenta en la USP-MADR no permite comparar el periodo anterior a la entrada en vigencia de la resolución 012/2007, puesto que se presenta una tendencia a la disminución de UFC entre ene-08 y feb-2012. Una posible explicación es la implementación de protocolos tales como los análisis de riesgos y puntos críticos de control (HACCP) y buenas prácticas ganaderas a los que la industria láctea no es ajena. Según Papademas y Bintsis (17) dichos protocolos se convierten en una solución costo efectiva para la obtención de un producto de mejor calidad, en la que se incluye la calidad higiénica. Igualmente, mejoras en la calidad pudieran estar asociadas a que el productor empieza a reconocer que ella está influenciando el valor pagado por litro de leche por parte del agente comprador, lo que se constituye en un estímulo para emprender acciones en este sentido.

Llama la atención el aumento de las UFC reportadas para la leche acopiada en la región 1 posterior a la entrada en vigencia de la resolución $468 / 2015$ y con ello el análisis de leche en laboratorios con acreditación en NTC-ISO/IEC 17025. Situación que amerita más estudios para ser explicada. La regresión cuantílica para las medianas de UFC en la región 2 no mostraron diferencias entre meses del año, resultados que contrastan con lo encontrado por Martínez et al (6) quienes evidenciaron diferencias en los reportes de UFC entre verano e invierno, siendo más altas en los primeros.

La calidad composicional de la leche acopiada en las regiones 1 y 2 cumplió con lo establecido en el decreto 616 de 2006 para todos los periodos (5). Sin embargo, se pudieron identificar diferencias en la calidad composicional e higiénica de la leche por regiones y por meses del año para los parámetros analizados. También se lograron identificar diferencias en porcentaje de grasa y proteína entre los periodos de observación para ambas regiones. Martínez et al (6) también identificaron diferencias en la calidad composicional de la leche según los meses del año, que estuvieron asociados con la frecuencia y distribución de las lluvias.

Las medianas de los porcentajes de proteína reportados para la región 2 calculadas en este estudio difieren de lo encontrado por Calderón et al (18) quienes reportaron promedios de $3.28 \%$ de proteína en leche acopiada de manera formal en Montería, Córdoba. Las diferencias pueden deberse a que en el presente estudio se contemplan resultados consolidados de toda la región 2, mientras Calderón et al (18) realizaron su investigación en ganaderías doble propósito del municipio de Montería.

La tendencia de estos resultados podría estar explicada por la estandarización de las mediciones a nivel de laboratorio, que permitió generar resultados precisos y acordes a la realidad del fenómeno. El estudio llevado a cabo por Khodabocus y Balgobin (19) demuestra que la acreditación de laboratorios en la norma ISO 
17025 genera resultados que son confiables y precisos dado que se obtienen parámetros similares a los obtenidos con las pruebas gold standard y con desviaciones estándar estrechas.

Los porcentajes de grasa reportados evidenciaron diferencias entre los periodos, particularmente en la región 1, donde se observó un aumento progresivo. Esto contrasta con lo evidenciado en la región 2, donde los porcentajes de grasa en leche disminuyeron a medida que avanzaron los periodos. Para el caso de la región 1, ese aumento progresivo en el contenido de grasa podría explicarse con los diferentes métodos de suplementación que han sido implementados en la región 1 como lo son las grasas protegidas que generan un mayor porcentaje de grasa en leche, selección de toros que trasmitan esta característica (20). El sistema de pago al productor asociado a calidad que se menciona en las normas ya descritas pudo incentivar mejoras en los sistemas de nutrición animal que influyen directamente en el contenido de grasa en leche, particularmente en la región 1 (21).

Respecto a las diferencias encontradas en las medianas de porcentaje de grasa para la región 2, donde a medida que avanzaban los periodos iba disminuyendo, podría deberse a la modificación de las diferentes técnicas de análisis en los laboratorios durante el periodo de estudio. Los resultados difieren de lo encontrado por Calderón et al (18) quienes reportaron porcentajes de grasa de 3,70\% para leche acopiada de manera formal en Montería, Córdoba para el año 2012 y de Arrieta et al (22) quienes encontraron un promedio de porcentaje de grasa de $4.95 \%$ en muestras de leche cruda comercializadas en municipios de Sucre.

El fuerte patrón estacional de la grasa reportada en la región 2 guarda relación con lo encontrado por otros autores, quienes identificaron la influencia que ejercen las variables meteorológicas sobre la producción y calidad de la leche. Esta influencia se identifica principalmente cuando las vacas son sometidas a condiciones ambientales desfavorables como altas temperaturas y baja precipitación, que genera una baja en la producción de leche y una menor calidad composicional (23). Sin embargo, estos hallazgos contrastan con lo reportado por Rodríguez-Rodríguez et al (24), quienes no encontraron diferencias para los porcentajes de grasa analizados según la época del año en muestras de leches cruda del sistema doble propósito en el departamento de Córdoba, Colombia.
Por último, los sólidos totales reportados para la leche acopiada, solo la región 1 mostró diferencias entre periodos. Se evidenció un aumento progresivo en los reportes, posiblemente asociados a mejoras en parámetros técnicos, productivos y administrativos que ejercen influencia sobre la calidad composicional de la leche, como lo son la genética, sanidad de hato y la nutrición de los animales (25). Para el caso de la región 2, no hubo diferencias en los reportes cuando se llevó a cabo la comparación por periodos.

Sin embargo, se evidenció una marcada estacionalidad en los porcentajes de sólidos totales reportados en la región 2 , similar a lo observado para grasa y proteína. Oliszewski et al (26) encontraron en Argentina que los sólidos totales varían significativamente durante el año encontrando los mayores niveles en otoño, comparado con invierno y primavera. Según Lambertz et al (15), la producción y calidad composicional de la leche se ven afectados por la interacción entre animales y diversas condiciones ambientales tales como temperaturas extremas.

Los resultados hallados en este estudio podrían estar asociados a la mayor exigencia que se hace a los laboratorios acreditados por parte de la ONAC para garantizar que la medición obtenida corresponda al valor real de la muestra motivo de análisis. Estas exigencias se ven reflejadas en el número de laboratorios que prestaban el servicio, puesto que se pasó de 108 laboratorios habilitados en abril 2012 a siete acreditados en NTC-ISO/IEC 17025 para diciembre 2020.

Se puede concluir que las diferencias encontradas sugieren que hubo una asociación entre la implementación de las normas emitidas inicialmente por el MADR para la habilitación de los laboratorios de análisis de calidad de leche, y luego la exigencia de la NTC-ISO/IEC 17025 para la acreditación de los laboratorios y la calidad higiénica y composicional de la leche acopiada de manera formal en Colombia. Sin embargo, esa asociación, no se vio reflejada de la misma manera en ambas regiones. En unos casos fue una influencia positiva que indicó una mejoría de los parámetros analizados, como el caso del porcentaje de grasa y proteína de la región 1 y las UFC en la región 2, pero en otros hubo diferencias significativas que evidenciaron detrimento de la calidad evaluada como las UFC de la región 1 en el tercer periodo. 
Con base en la información analizada se concluye que es necesario realizar análisis más exhaustivos respecto a las razones por las cuales se dan las diferencias en calidad composicional e higiénica de la leche identificadas. Además, sería útil conocer las razones por las cuales las diferencias identificadas fueron más evidentes en la región 1.

\section{Conflicto de intereses}

Los autores de este estudio manifiestan que no existe conflicto de intereses con la publicación de este manuscrito.

\section{Agradecimientos}

Al Sistema General de Regalias (SGR), Departamento de Antioquia a través de su Secretaria de Agricultura y Desarrollo Rural y MINCIENCIAS por su apoyo o cofinanciación del proyecto "Fortalecimiento empresarial de los productores de leche en el departamento de Antioquia mediante la mejora de la calidad e inocuidad de la leche a través de la generación y el uso y evaluación de un producto de desarrollo tecnológico" contrato No 80740-013-2019, en el marco del cual se generó esta publicación.

\section{REFERENCIAS}

1. USP-MADR. Precio por litro de leche pagado al productor a nivel nacional y regional. Unidad de Segumiento de Precios de la Leche. Ministerio de Agricultura y Desarrollo Rural: Colombia; 2020. http://uspleche. minagricultura.gov.co/precio-pagado-alproveedor-con-bonificaciones-voluntariasnacional-y-regional.html

2. Restrepo-Betancur LF, Peña-Serna C, Zapata-López N, Restrepo-Betancur LF. Disponibilidad de Leche de los Países Sudamericanos en las Últimas Cinco Décadas: Elementos para Análisis y Perspectivas Futuras. Inf tecnol. 2019; 30(4):77-84. http://hdl.handle.net/10495/12387

3. Gaona RC, Terranova MV, Hernández E, Alegría KG, Benavides RM, Guerrero HS, et al. Genetic improvement in dairy cows. The essence of true animal production. Acta Agron. 2016; 64(3):296-306. https:// revistas.unal.edu.co/index.php/acta agronomica/article/view/50263/56012

4. Vásquez JF, Loaiza ET, Olivera M. Calidad higiénica y sanitaria de leche cruda acopiada en diferentes regiones colombianas. Orinoquia $2012 ; 16(2): 13$. https:// orinoquia.unillanos.edu.co/index.php/ orinoquia/rt/printerFriendly/251/737

5. Decreto 616. Por el cual se expide el Reglamento Técnico sobre los requisitos que debe cumplir la leche para el consumo humano. Ministerio de la Protección Social: Colombia; 2006. https://www.ica.gov.co/ getattachment/15425e0f-81fb-4111-b21563e61e9e9130/2006D616.aspx
6. Martínez M, Gómez C. Calidad composicional e higiénica de la leche cruda recibida en industrias lácteas de Sucre, Colombia. Biotecnología En El Sector Agropecuario Y Agroindustrial. 2013; 11(2):93-100. https://revistas.unicauca.edu.co/index. php/biotecnologia/article/view/309

7. Rodríguez RV, Calderón RA, Vergara GO. Calidad de leches crudas en tres empresas acopiadoras en Córdoba. Rev Colomb Cienc Anim RECIA. 2014; 6(1):103-15. https:// doi.org/10.24188/recia.v6.n1.2014.222

8. D'Amico DJ, Donnelly CW. Microbiological quality of raw milk used for small-scale artisan cheese production in Vermont: Effect of farm characteristics and practices. J Dairy Sci. 2010; 93(1):134-147. https:// doi.org/10.3168/jds.2009-2426

9. Smigic N, Djekic I, Tomasevic I, Miocinovic J, Gvozdenovic R. Implication of food safety measures on microbiological quality of raw and pasteurized milk. Food Control. 2012;25(2):728-31. https://doi. org/10.1016/j.foodcont.2011.12.022

10. Resolución 017. Por el cual se establece el sistema de pago de leche cruda al proveedor. Ministerio de Agricultura y Desarrollo Rural: Colombia; 2012. [Consultado octubre de 2019]. https:// www.icbf.gov.co/cargues/avance/docs/ resolucion minagricultura 0017 2012.htm 
11. Resolución 468. Por la cual se modifica la resolución 017 de 2012, modificada por la resolución 077 de 2015. Ministerio de Agricultura y Desarrollo Rural: Colombia; 2015. [Consultado octubre de 2019]. https:// www.icbf.gov.co/cargues/avance/docs/ resolucion minagricultura 0468 2015.htm

12. Norma técnica NTC-ISO/IEC Colombiana 17025. Requisitos generales para la competencia de los laboratorios de ensayo y calibración. Instituto Colombiano de Normas Técnicas (ICONTEC): Colombia; 2005. [Consultado diciembre 2019]. https://www.invima.gov.co/images/pdf/ red-nal-laboratorios/resoluciones/NTC-ISOIEC 17025-2005.pdf

13. Organismo Nacional de Acreditación - ONAC. Directorio de laboratorios acreditados: Colombia; 2020 [Consultado 10 de agosto 2020]. https://onac.org.co/directorio-deacreditados

14. Koenker R. Quantile regression. New York. Cambridge University Press. 2005. https:// books.google.com.co/books?id=hdkt7V4NX $\underline{\text { sgC\&printsec }=\text { frontcover } \& \text { hl }=\text { es } \& \text { source }=\mathrm{g}}$ bs ge summary $r \& c a d=0 \# v=$ onepage $\& q \&$ $\underline{\mathrm{f}=\text { false }}$

15. Lambertz C, Sanker C, Gauly M. Climatic effects on milk production traits and somatic cell score in lactating Holstein-Friesian cows in different housing systems. J Dairy Sci. 2014;97(1):319-329. http://dx.doi. org/10.3168/jds.2013-7217

16. Hoye A. Recommend or mandate? A systematic review and meta-analysis of the effects of mandatory bicycle helmet legislation. Accid Anal Prev. 2018; 120:239-249. https://doi.org/10.1016/j. aap.2018.08.001

17. Papademas $P$, Bintsis $T$. Food safety management systems (FSMS) in the dairy industry: A review. Int J Dairy Technol. 2010；63(4):489-503. http://dx.doi. org/10.1111/j.1471-0307.2010.00620.x

18. Calderón A, Rodríguez V, Arrieta G, Martínez $\mathrm{N}$, Vergara $\mathrm{O}$. Calidad fisicoquímica y microbiológica de leches crudas en empresas ganaderas del sistema doble propósito en Montería (Córdoba), Rev UDCA Act \& Div Cient. 2012; 15(2):399-407. https:// revistas.udca.edu.co/index.php/ruadc/ article/view/841
19. Khodabocus F, Balgobin K. Implementation and Practical Benefits of ISO/IEC 17025:2005 in a Testing Laboratory. Univ Mauritius Res J. 2011;17(1):27-60. http://dx.doi. org/10.4314/umrj.v17i1.70730

20. Duarte V. J, Ramírez Z. G, Castañeda S. R. Grasa sobrepasante: Aplicaciones y su proceso de obtención para la alimentación de rumiantes en el trópico. Rev Colomb Cienc Anim - RECIA. 2016; 8(2):228. https://doi. org/10.24188/recia.v8.n2.2016.192

21. García C, Montiel R, Borderas Y. Grasa y proteína de la leche de vaca: componentes, síntesis y modificación. Arch Zootec. 2014; 63:85-105. https://doi.org/10.21071/ az.v63i241.592

22. Arrieta B, Gomezcaceres P, Albis F, CalderónRangel A, Rodríguez R. Calidad de la leche cruda para consumo humano en dos localidades de Sucre (Colombia). Rev MVZ Córdoba 2019; 24(3):7355-7361. https:// doi.org/10.21897/rmvz.1829

23. Hill DL, Wall E. Dairy cattle in a temperate climate: The effects of weather on milk yield and composition depend on management. Animal. 2014; 9(1):138-149. http://dx.doi. org/10.1017/S1751731114002456

24. Rodríguez-Rodríguez $\mathrm{V}$, Acosta-Ruiz $\mathrm{A}$. Calidad de leches crudas en sistemas doble propósito en Córdoba (Colombia), en condiciones de máxima y mínima precipitación. Rev Vet Zootec. 2014; 8(2):7286. http://190.15.17.25/vetzootec/index. $\mathrm{php} /$ component/content/article?id $=104$

25. Escoto FC, Vargas AC, Oño IM. La calidad estándar de la leche en el estado de Hidalgo, México. Rev Mex Cienc Pecu. 2013;4(1):82. https://cienciaspecuarias.inifap.gob. mx/ index.php/Pecuarias/article/view/2827

26. Oliszewski R, Cisint J, Medina CF. Caracterización composicional, fisicoquímica y microbiológica de leche de vaca de la cuenca de Trancas. COCINET. 2016; 36(4105):31-9. https://www. conicet. gov.ar/new scp/detalle.php?keywords $=\&$ $\underline{i d=31611 \& \text { articulos }=\text { yes } \& \text { detalles }=\text { yes } \& a}$ rt id $=6712611$ 